\title{
The effect of stimulus availability on task choice in voluntary task switching
}

\author{
CATHerine M. ARrington \\ Lehigh University, Bethlehem, Pennsylvania
}

\begin{abstract}
The voluntary task switching paradigm allows subjects to choose which task to perform on each trial in a stimulus environment affording multiple tasks. The present study examined the effect of stimulus availability on task choice. Subjects viewed displays containing a digit and a letter and performed either an even/odd or a consonant/vowel judgment on each trial. The target stimuli appeared with a stimulus onset asynchrony (SOA) of $0,50,100$, or $150 \mathrm{msec}$. The probability of performing the task associated with Stimulus 1 increased as SOA increased, indicating an effect of external or stimulus-driven factors on task choice. This effect of stimulus availability on task choice was greater when the response-stimulus interval was $400 \mathrm{msec}$ than when it was $2,000 \mathrm{msec}$. This interaction of preparation interval and stimulus availability is explained within a model of task choice that includes both internal processes and external influences.
\end{abstract}

Task switching procedures have become widely used in studying cognitive control processes (Logan, 2003; Monsell, 2003). Standard task switching paradigms, such as alternating runs or explicit task cuing, specify the task to be performed on each trial and measure task performance when the task repeats versus when the task switches (Meiran, 1996; Rogers \& Monsell, 1995). Reaction times (RTs) are slower on task switches than on task repetitions, and these switch costs are taken to reflect control processes engaged when task sets change (Rogers \& Monsell, 1995) or disruptions of task performance on task switches due to carryover of previous task sets (Allport \& Wylie, 2000). However, a full understanding of cognitive control in multitask environments must involve examination of not only sequential task performance, but also task selection.

Consider real-world multitask environments: Individuals in situations that allow for several actions may perform tasks in various equally valid sequences and must choose how to sequence their behavior. To address this difference between natural and experimental environments, $\mathrm{Ar}$ rington and Logan $(2004,2005)$ recently developed a voluntary task switching procedure in which subjects choose which one of two or more possible tasks to perform on each trial. Subjects are instructed to perform the tasks equally often and in a random order but are not provided with instructions about which task to perform on a given trial. As in other task switching procedures, performance differences on task repetitions and task switches show robust switch costs. In addition, the voluntary task switching procedure allows for another performance measure: task choice. The probabilities with which subjects carry out specific tasks and transition among tasks provide a new avenue for investigating the control processes involved in multitask performance. Unlike switch cost measures that assess processes influencing performance, task choice measures provide insight into the processes influencing selection of a task to perform. The question of interest becomes not only how but also why a task is performed.

Arrington and Logan $(2004,2005)$ demonstrated several basic characteristics of task choice in previous voluntary task switching studies. In general, subjects complied well with instructions to perform tasks (e.g., parity and magnitude judgments of single digits) equally often. However, subjects showed a repetition bias, performing task repetitions more frequently than would be expected if tasks were performed in a random sequence. This repetition bias was greatest when the response-stimulus interval (RSI) was short, and it decreased as RSI increased. At longer RSIs, subjects may be better able to carry out controlled processes involved in task choice, which thus reduces the likelihood of simply repeating the task. Finally, task choice appeared for the most part to be unaffected by external stimulus characteristics such as target identity. However, there was evidence that influences of external stimuli on task choice could be found in some cases. In only two of five experiments from Arrington and Logan (2005) were subjects slightly more likely to choose to repeat a task if the stimulus repeated. On the basis of the small and inconsistent nature of these results across experiments, they concluded that task choice in voluntary task switching environments was largely controlled in a top-down fashion, thus providing evidence for voluntary behavior that could be measured within this experimental paradigm.

Mayr and Bell (2006) recently challenged this conclusion in an investigation of factors influencing choice in voluntary task switching. In the context of a larger consid-

C. M. Arrington, kate.arrington@lehigh.edu 
eration of individual differences in voluntary task switching performance, they examined the effect of target repetition on task choice. Across two different pairs of tasks, performance of task repetitions was significantly more frequent when the target stimulus repeated. This effect was particularly strong in spatial mapping tasks that involved only two target stimuli, thus allowing for frequent target repetitions. These findings led Mayr and Bell to conclude that "the cognitive system is highly susceptible to exogenous influences during voluntary choice" (p. 779). These early findings raise questions about the degree to which task choice in voluntary task switching experiments reflects voluntary behavior.

In voluntary task switching, task choice is defined on the basis of the response performed on each trial (but see Arrington \& Logan, 2005, Experiment 6, for a method for directly measuring task choice). The task performed may be determined by multiple factors; however, these factors must be reflected in the task set that ultimately leads to a response. The term task set has been used in at least two ways in the task switching literature: (1) to describe a state of preparation for a particular task, and (2) to specify a group of component operations needed to perform a particular task. In the present context, the second definition may provide the greatest traction for operationalizing task choice. Logan and Gordon's (2001) executive control of Bundesen's theory of visual attention (ECTVA) provides one of the more explicit definitions of a task set as "the set of control parameters that is necessary to program TVA to perform a particular task" (p. 396). Furthermore, ECTVA proposes two levels of task representation in working memory: task level and parameter level. The task-level representation involves the task instructions that may be held in a propositional representation. The task-level representation may be accessible to conscious awareness and may loosely be thought of as task goals. Once a task-level representation is formed, a parameter-level representation is derived and transmitted from working memory to the subordinate systems that carry out the tasks. It is this parameter-level representation that determines the task set and ultimately what response is performed on a given trial. In voluntary task switching, the operationalization of task choice based on the response performed means that task choice is more tightly linked to the parameter level, which guides task execution, rather than the task level, which may be more closely associated with intentional choice.

ECTVA views attention as a choice process, a race among all possible categorizations. The speed with which the race is run depends on the parameters for the given task sets. In ECTVA's simplest form, the probability of a categorization is determined by the external evidence for that categorization, $\eta$, and by the internal bias toward making a particular categorization, $\beta$. Within ECTVA, the $\eta$ values are determined by the external stimulus environment, and the $\beta$ values are under the control of the executive. Together, they represent a critical part of the task set necessary for carrying out a particular task. Thus, the determination of which task will be performed results from a combination of factors, some of which are actively set by the executive and some of which are passively dictated by the stimulus envi- ronment. Under this view, task choice is driven by many factors. It is the goal of the present research to specify some of these factors and describe their relationships.

In real-world multitask environments, many external factors may influence the choice that an individual makes about which task to perform at a given time. One of these factors is the availability of the stimulus affording a task. Stimulus availability may be highly dynamic for a variety of reasons. As objects move, or as individuals move through an environment, stimuli may be occluded from view or shifted to an inaccessible location. More basically, shifts in gaze and focus of visual attention result in constant updating of the external stimuli currently available to the cognitive system. The present research investigates whether such stimulus availability influences task choice in multitask environments. On the one hand, if task choice is driven strictly in a top-down fashion on the basis of internal goals (in a task-level representation), then stimulus availability should not influence task choice. In this view, the selection of a task to perform occurs at a stage independent of and prior to the processing of stimuli by the cognitive system once the appropriate task set is established. On the other hand, if task choice is influenced by both top-down and bottom-up factors (in a parameterlevel representation), then stimulus availability should influence task choice. The availability of a stimulus to the cognitive system may initiate processing of that stimulus or performance of a task afforded by that stimulus (Norman \& Shallice, 1986). In multitask environments where two or more task sets may be maintained simultaneously in working memory, greater availability of the stimulus associated with one of those task sets may influence subjects to perform that task with higher probability.

\section{EXPERIMENT 1}

In the present experiments, stimulus availability was manipulated by varying the time at which the stimuli affording each task were made available to the subjects. Subjects performed two tasks: digit classification (i.e., even/odd) and letter classification (i.e., consonant/vowel). Both a number and a letter appeared on every trial, allowing for presentation of the two stimuli with variable stimulus onset asynchrony (SOA). If the availability of external stimuli does influence task choice, then the probability of performing the task associated with the first stimulus to appear should increase as the SOA between the two stimuli increases.

\section{Method}

Subjects. Sixteen Lehigh University undergraduates participated in exchange for partial course credit. All subjects reported normal or corrected-to-normal visual acuity.

Apparatus and Stimuli. Experimental testing was carried out on a Dell Dimension with a 17-in. CRT monitor. The display of stimuli and recording of responses were controlled using the E-Prime software package.

All stimuli were presented in black Courier New font on a light gray background. Viewing distance was not constrained. The fixation screen contained a $5 \times 5 \mathrm{~mm}$ cross in the center of the screen and four $7 \times 5 \mathrm{~mm}$ pound signs (\#) serving as placeholders at the possible stimulus locations. The placeholders were positioned at the apices of an invisible square, each centered $20 \mathrm{~mm}$ from fixation. 
The target stimuli for the even/odd task were the numbers 2-9 and for the consonant/vowel task were the uppercase letters A, B, C, E, $\mathrm{I}, \mathrm{L}, \mathrm{U}$, and $\mathrm{W}$.

Procedure. Trials began with the onset of the fixation screen containing the fixation cross and placeholders for $1,000 \mathrm{msec}$. Then Stimulus 1 (S1) appeared, replacing one of the four placeholders. Following the SOA of 0, 50,100, or $150 \mathrm{msec}$, manipulated within blocks, Stimulus 2 (S2) appeared at another of the placeholder positions. S1 was chosen from the letter and number tasks equally often. The identity and position of each stimulus were determined at random. Both stimuli and the remaining placeholders stayed on the screen until a keypress response was performed. Subjects responded using the index and middle fingers of both hands on the home keys of the keyboard. Responses for a given task were made with the same hand, and the specific S-R mappings were counterbalanced across subjects. The univalent responses allowed for classification of the task performed by the subject. Subjects completed practice blocks of 16 trials for each task and then received standard voluntary task switching instructions modeled after those published elsewhere ( $\mathrm{Ar}-$ rington \& Logan, 2004): Subjects were instructed to perform each task equally often and in a random order. Subjects were not explicitly informed that the two stimuli might appear at different times and no further instructions regarding how to select which task to perform were provided. Following a block of practice with the voluntary task switching procedure, subjects completed 8 blocks of 64 trials.

\section{Results and Discussion}

Trials were sorted into task conditions based on the hand with which the response was performed on trial $n$ and into task transition conditions based on the task performed on trials $n$ and $n-1$. Overall accuracy was high, $97.7 \%$. Trials on which subjects committed an error and the subsequent trial were eliminated for all analyses. For the RT analyses, all trials on which the RT was less than 150 or greater than $3,000 \mathrm{msec}$ were trimmed. RT trimming resulted in the removal of $2.1 \%$ of the trials. RTs were calculated from the onset of the stimulus on which the task was performed, either S1 or S2. Significance testing was performed with $\alpha=.05$.

Overall, subjects performed the two tasks equally often with a mean proportion of trials on which the letter task was performed of .506, which did not differ from the instructed value of $.5[t(15)=1.1, p>.1]$. The analysis of task transition showed that subjects chose to repeat tasks slightly more frequently than expected by chance, .536 ; however, this value did not differ statistically from .5 $[t(15)=1.2, p>.1]$. This result is in line with previous voluntary task switching experiments where the repetition bias was small and did not differ from chance at longer RSIs such as that used in this experiment. ${ }^{1}$ Taken together, these two analyses indicate that subjects were largely able to perform in accordance with the stated instructions.

Task performance measured in terms of RT showed standard switch costs $(M=157 \mathrm{msec})$, with task repetitions being performed more quickly than task switches. Responses were slightly slower when the task performed was associated with $\mathrm{S} 1$ than with $\mathrm{S} 2(M \mathrm{~s}=1,050$ and 1,019 msec, respectively). Keeping in mind that the RTs were calculated from the onset of the stimulus on which the task was performed, this pattern is likely due to responding to S2 being less influenced by the presence of the other stimulus that onset before the RT interval for $\mathrm{S} 2$ responses began. Mean RTs for all cells in the de-
Table 1

Reaction Times (in Milliseconds) for Task Repetitions and Task Switches, As a Function of Stimulus Onset Asynchrony (SOA, in Milliseconds) and Stimulus on Which the Task Was Performed for Experiment 1

\begin{tabular}{|c|c|c|c|c|c|c|c|c|}
\hline & \multicolumn{8}{|c|}{ SOA } \\
\hline & \multicolumn{2}{|c|}{0} & \multicolumn{2}{|c|}{50} & \multicolumn{2}{|c|}{100} & \multicolumn{2}{|c|}{150} \\
\hline & $M$ & $S E$ & $M$ & $S E$ & $M$ & $S E$ & $M$ & $S E$ \\
\hline \multicolumn{9}{|l|}{ Response to S1 } \\
\hline Task repetitions & 945 & 49 & 957 & 56 & 1,016 & 63 & 980 & 55 \\
\hline Task switches & 1,127 & 51 & 1,128 & 50 & 1,106 & 62 & 1,143 & 67 \\
\hline \multicolumn{9}{|l|}{ Response to S2 } \\
\hline Task repetitions & 939 & 46 & 946 & 51 & 940 & 54 & 927 & 55 \\
\hline Task switches & 1,107 & 65 & 1,103 & 59 & 1,105 & 63 & 1,085 & 78 \\
\hline
\end{tabular}

sign appear in Table 1. A 2 (task transition: repetition or switch) $\times 2$ (stimulus on which the task was performed: $\mathrm{S} 1$ or S2) $\times 4(\mathrm{SOA}: 0,50,100$, or $150 \mathrm{msec})$ repeated measures ANOVA conducted on mean RTs showed significant main effects of task transition $[F(1,15)=60.1$, $\left.M S_{\mathrm{e}}=26,012.5\right]$ and stimulus $\left[F(1,15)=5.9, M S_{\mathrm{e}}=\right.$ 10,697.1]. No other effects were significant.

Of primary interest in the present experiment was the effect of stimulus availability on task choice, measured in terms of the probability of performing the task associated with $\mathrm{S} 1$, or $p(\mathrm{~S} 1)$. As can be seen in Figure 1, $p(\mathrm{~S} 1)$ values increased with increasing SOA between $\mathrm{S} 1$ and $\mathrm{S} 2$. The linear trend of SOA was significant $\left[F(1,15)=10.2, M S_{\mathrm{e}}=\right.$ $0.009]$. Across SOA the $p(\mathrm{~S} 1)$ values were significantly different from chance only for the two longer intervals. Examination at the level of individual subjects revealed that 13 of 16 showed a positive slope in $p(\mathrm{~S} 1)$ values across SOA and that average $p(\mathrm{~S} 1)$ values varied between .47 and .75. Thus the effect of SOA on task choice was both robust across subjects and small in size, ruling out the possibility that subjects used an explicit strategy to perform the task associated with $\mathrm{S} 1$ in order to speed responding.

These results demonstrate a significant relationship between stimulus availability and task choice in voluntary

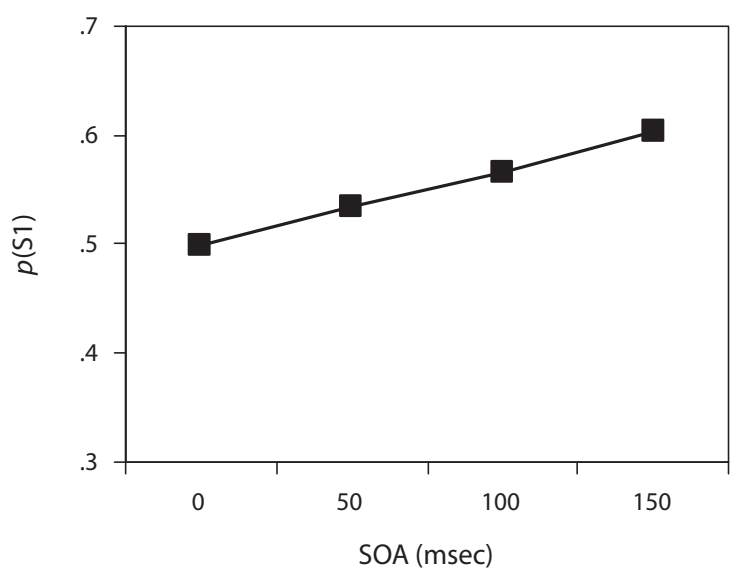

Figure 1. Proportion of trials on which the task associated with $\mathrm{S} 1$ was performed, as a function of the stimulus onset asynchrony (SOA) between S1 and S2 for Experiment 1. 
task switching environments. This finding provides clearer evidence of the influence of external factors on task choice than has been obtained in the previous analyses performed by Arrington and Logan (2005) and Mayr and Bell (2006), who looked at the effect of stimulus repetition on the choice to repeat or switch tasks. In the present experiment, the parametric manipulation of SOA showed a highly linear relationship between stimulus availability and subjects' likelihood of performing a task. However, it is important to note that even at the longest SOA, the average $p(\mathrm{~S} 1)$ value was .6, suggesting that task choice is largely influenced by other factors. In addition, examination of the task transition effects showed that subjects' choices of task were not significantly different from chance in performance of task repetitions versus task switches. This finding suggests that subjects may be controlling task choice sufficiently to meet the instructions to perform tasks in a random order. These results suggest that both internal goal-driven and external stimulus-driven factors influence task choice. The present experiment systematically manipulated only the stimulus environment and was not designed to manipulate internal control, thus prohibiting the examination of any interaction between these factors.

\section{EXPERIMENT 2}

Experiment 2 examined the relationship between internal and external factors affecting task choice. The procedure was the same as in Experiment 1, except for an additional manipulation of RSI to include short and long intervals between tasks. Manipulations of RSI influence task transition probabilities, with the repetition bias being greater at shorter RSIs (Arrington \& Logan, 2004). Many models of task switching performance have proposed that the time between trials serves as a preparation interval (Meiran, 1996; Rogers \& Monsell, 1995; cf. Logan, 2003, for an alternative interpretation of the time-course effects). If during the RSI subjects are preparing for the upcoming task, preparation during voluntary task switching is likely to involve internal selection of the task goal for the upcoming trial. In the framework of ECTVA (Logan \& Gordon, 2001), this situation would correspond to both establishing the task-level representation, or task goal, and subsequently transmitting the parameterlevel representation to the subordinate systems. For shorter RSIs, there will be a larger proportion of trials on which this task selection process is incomplete when the stimuli appear, either because the individual has not formed an intention to perform a particular task or because the task set has not been established. This incomplete preparation on trials with shorter RSIs may lead to greater effects of external stimuli than on trials with longer RSIs, when subjects more often complete an act of internal task selection before the stimuli appear. If such a relationship exists between internal and external factors influencing task choice, the effect of SOA should interact with the manipulation of RSI.

\section{Method}

Subjects. Twenty-four individuals from the Lehigh University community participated for either partial course credit or $\$ 10$. All subjects reported normal or corrected-to-normal visual acuity.
Stimuli, Apparatus, and Procedure. The tasks and experimental setup were identical to those of Experiment 1, except for the inclusion of a manipulation of RSI. The RSIs were 400 and 2,000 msec, thus bracketing the 1,000-msec RSI used in Experiment 1. RSI was manipulated within blocks. Subjects performed 14 blocks of 64 trials.

\section{Results and Discussion}

The data coding and trimming procedures were the same as in Experiment 1. Overall accuracy was $97.7 \%$. RT trimming resulted in the removal of less than $1 \%$ of the trials. Again, subjects performed the two tasks equally often, with a mean proportion of trials on which the letter task was performed of .507, which did not differ from the instructed value of $.5[t(23)=1.7, p=.1]$. Examination of the task transition probabilities showed a repetition bias at the short RSI but not at the long RSI, with repetition probabilities of .610 and .499 , respectively. The effect of RSI on transition probabilities was significant $[t(23)=9.2]$. This finding replicates results from Arrington and Logan $(2004,2005)$.

The effect of task transition on RTs also replicated the standard preparation effect on switch costs. The switch costs were greater at the short RSI $(M=171 \mathrm{msec})$ than at the long RSI $(M=74 \mathrm{msec})$. The $157-\mathrm{msec}$ switch cost found in Experiment 1 was similar in magnitude to the short RSI in the present experiment, suggesting that when subjects were exposed to only one RSI, they may have been "lazy" in preparing for a task switch (Altmann, 2004). Table 2 provides RT data for each cell in the design. A 2 (RSI: 400 or $2,000 \mathrm{msec}) \times 2$ (task transition: repetition or switch) $\times 2$ (stimulus on which the task was performed: S1 or S2) $\times 4$ (SOA: $0,50,100$, or $150 \mathrm{msec}$ ) repeated measures ANOVA performed on the mean RTs showed significant main effects of RSI $\left[F(1,23)=8.28, M S_{\mathrm{e}}=18,317.1\right]$ and task transition $\left[F(1,23)=87.3, M S_{\mathrm{e}}=33,145.8\right]$, as well as a significant interaction of these factors capturing the abovenoted effect of preparation on switch costs $[F(1,23)=$ 38.2, $\left.M S_{\mathrm{e}}=11,808.2\right]$. Unlike in Experiment 1, the RT was influenced by SOA, which showed a significant main effect $\left[F(3,69)=5.9, M S_{\mathrm{e}}=5,441.4\right]$ and interacted significantly with stimulus on which the task was performed $\left[F(3,69)=4.22, M S_{\mathrm{e}}=8,302.7\right]$ and with task transition $\left[F(3,69)=4.43, M S_{\mathrm{e}}=5,177.8\right]$. RTs were stable across SOA when the task was performed on S1 and decreased monotonically across SOA when the task was performed on S2. This decrease over SOA may have occurred because as the separation between S1 and S2 increased, the disruption in processing of $\mathrm{S} 2$ as a result of interference from $\mathrm{S} 1$ decreased. S1 processing would not see a similar change in RT over SOA, because S2 would always be appearing during the response interval for responses to S1. RTs were stable across SOA for task repetitions and decreased monotonically across SOA for task switches. The finding that SOA influenced RT only on switch trials may be indicative of a weaker task set on these trials, which would allow for more disruption of stimulus processing when the two stimuli appear at the same time. However, similar analyses in Experiment 1 failed to show any significant effects involving SOA, making the present interpretation subject to further investigation (see Yeung \& Monsell, 2003, for further consideration of how RT switch costs are influenced 
Table 2

Reaction Times (in Milliseconds) for Task Repetitions and Task Switches, As a Function of Response-Stimulus Interval (RSI, in Milliseconds), Stimulus Onset Asynchrony (SOA, in Milliseconds), and Stimulus on Which the Task Was Performed for Experiment 2

\begin{tabular}{|c|c|c|c|c|c|c|c|c|c|}
\hline & \multirow[b]{3}{*}{ RSI } & \multicolumn{8}{|c|}{ SOA } \\
\hline & & \multicolumn{2}{|c|}{0} & \multicolumn{2}{|c|}{50} & \multicolumn{2}{|c|}{100} & \multicolumn{2}{|c|}{150} \\
\hline & & $M$ & $S E$ & $M$ & $S E$ & $M$ & $S E$ & $M$ & $S E$ \\
\hline \multicolumn{10}{|l|}{ Response to S1 } \\
\hline Task repetitions & 400 & 840 & 26 & 866 & 29 & 857 & 30 & 874 & 29 \\
\hline Task switches & 400 & 1,037 & 39 & 1,017 & 31 & 1,060 & 40 & 1,032 & 38 \\
\hline Task repetitions & 2,000 & 949 & 40 & 938 & 39 & 938 & 40 & 960 & 35 \\
\hline Task switches & 2,000 & 1,043 & 36 & 1,007 & 35 & 1,025 & 42 & 979 & 30 \\
\hline \multicolumn{10}{|l|}{ Response to S2 } \\
\hline Task repetitions & 400 & 849 & 28 & 835 & 26 & 811 & 26 & 832 & 25 \\
\hline Task switches & 400 & 1,044 & 39 & 1,008 & 40 & 976 & 41 & 961 & 36 \\
\hline Task repetitions & 2,000 & 925 & 31 & 911 & 34 & 877 & 28 & 880 & 32 \\
\hline Task switches & 2,000 & 1,012 & 34 & 1,000 & 41 & 959 & 34 & 946 & 37 \\
\hline
\end{tabular}

by relative interference between tasks that is manipulated by temporal asynchronies in stimulus onset).

Figure 2 shows the average $p(\mathrm{~S} 1)$ values as a function of SOA, separated by RSI. The effect of SOA replicated the results of Experiment 1, showing the influence of external stimulus availability on task choice: As SOA increased, the $p(\mathrm{~S} 1)$ values also increased. RSI, manipulating the time to prepare for the upcoming trial, had the opposite effect: As RSI increased, the $p(\mathrm{~S} 1)$ values decreased. Importantly, the SOA and RSI variables interacted in such a way that the effect of SOA was smaller when the RSI was longer. A 2 (RSI: 400 or $2,000 \mathrm{msec}) \times 4(\mathrm{SOA}: 0,50,100$, or $150 \mathrm{msec}) \mathrm{re}-$ peated measures ANOVA confirmed the significant linear trend in $p(\mathrm{~S} 1)$ as a function of SOA $\left[F(1,23)=9.2, M S_{\mathrm{e}}=\right.$ $0.011]$ and a main effect of RSI $\left[F(1,23)=9.7, M S_{\mathrm{e}}=\right.$ $0.002]$. The interaction of RSI with the linear trend of the SOA effect was also significant $\left[F(1,23)=5.8, M S_{\mathrm{e}}=\right.$ $0.001]$. This analysis captures the increasing separation between the short and long RSI conditions as SOA increases. At the level of individual subjects, the 19 of 24 (400-msec $\mathrm{RSI})$ and 16 of 24 (2,000-msec RSI) displayed a positive

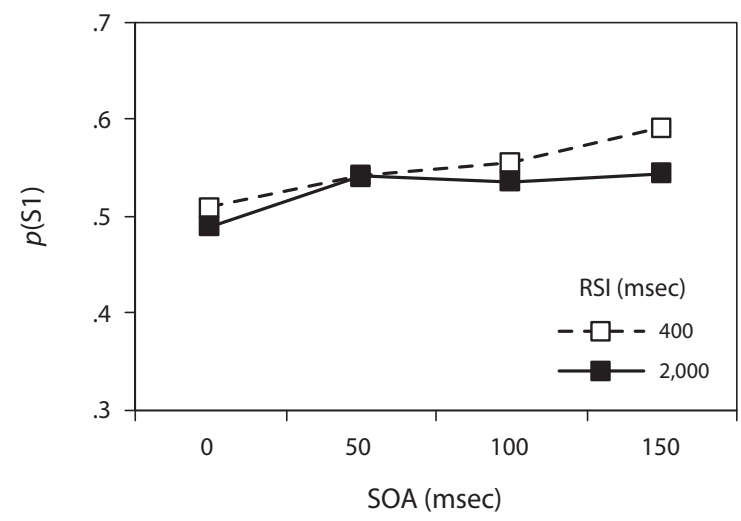

Figure 2. Proportion of trials on which the task associated with S1 was performed, as a function of the stimulus onset asynchrony (SOA) between $\mathrm{S} 1$ and $\mathrm{S} 2$ and separated by response-stimulus interval (RSI), for Experiment 2. slope for $p(\mathrm{~S} 1)$ values as a function of SOA, and average $p(\mathrm{~S} 1)$ values varied between .49 and $.65(400-\mathrm{msec} \mathrm{RSI})$ and .46 and $.63(2,000-\mathrm{msec}$ RSI).

The key result of Experiment 2 is the demonstration that the effect of stimulus availability on task choice is reduced when subjects have more time to prepare for the upcoming task. This finding is appealingly simple: If there has not been adequate time to form an internal task goal before the stimuli appear, then relative stimulus availability has a larger influence on task choice, pushing subjects toward more frequent performance of the task associated with S1. However, if there has been adequate time to form a task goal and establish a task set, then subjects are less influenced by stimulus availability. Once an internal task goal has been established, subjects may search for the target stimulus that affords the chosen task regardless of when it becomes available. This explanation suggests that task goals may serve as a guide for selection of task-relevant external informationan idea similar to the concept of attentional control settings, which has been studied extensively in the visual attention literature (Folk, Remington, \& Johnston, 1992).

These results provide a potential explanation for the different conclusions drawn in previous considerations of the role of external stimuli in directing task choice in voluntary task switching. Arrington and Logan (2005) found relatively weak evidence for the influence of external stimuli, whereas Mayr and Bell (2006) found much larger effects of stimulus repetition on task choice. Mayr and Bell used a 100 -msec RSI, allowing very little time for subjects to form an internal task goal prior to the onset of the target stimulus. On the other hand, Arrington and Logan (2005) examined the effect of stimulus repetition over a range of RSIs from 100 to $1,000 \mathrm{msec}$. The variability in RSI, as well as the longer time intervals, allowing for more internal control over task choice, may have led to smaller effects of stimulus repetition in these experiments.

\section{GENERAL DISCUSSION}

The results of two experiments demonstrated an influence of stimulus availability on task choice. In a voluntary 
task switching environment where two stimuli appeared with varying SOA, performance of the task associated with the first stimulus to appear increased as the SOA between the two stimuli increased. This effect of external stimulus availability was reduced when subjects had longer to prepare for the upcoming trial. The findings from these two experiments demonstrate effects of both external stimuli and internal preparation on task choice in multitask environments.

The combination of external, or stimulus-driven, and internal, or goal-directed, influences on behavior has been studied in varied cognitive domains, such as visual attention (Yantis \& Jonides, 1990) and attention to action (Norman \& Shallice, 1986). These two factors are considered prominently in theorizing about performance in standard task switching environments. Numerous researchers have proposed mechanisms underlying switch costs caused by external factors (Allport \& Wylie, 2000; Waszak, Hommel, \& Allport, 2003), internal factors (Meiran, 1996), or both (Rogers \& Monsell, 1995; Ruthruff, Remington, \& Johnston, 2001; for recent reviews, see Logan, 2003; Monsell, 2003). Although the specific mechanisms proposed in these studies differ, the focus has been on explaining the slower and less accurate performance that occurs on task switches relative to task repetitions. The present research examined the influence of internal and external factors on task choice as well as on task performance.

Arrington and Logan (2005) argued that the voluntary task switching procedure must necessarily involve internal control of task choice, because, unlike in other task switching paradigms, the task identity is not cued by the experimenter. Furthermore, they suggested that choice probabilities may involve a competition between a representativeness heuristic and an availability heuristic. The representativeness heuristic involves comparing the previous $n$ trials in working memory with an internal concept of a random sequence and selecting the next task in order to make the series most representative of this concept of randomness (Rapoport \& Budescu, 1997). The availability heuristic results in task choice driven by habitual or more easily retrieved responses (Baddeley, Chincotta, \& Adlam, 2001). The availability heuristic may result in a repetition bias due to the greater availability of the recently performed task set. The present findings may be reconciled to the description of Arrington and Logan (2005) in the following way. The representativeness heuristic requires consideration of the recent trials in working memory and matching the current trial sequence to the internal representation of a random sequence. It takes time and requires internal control to carry out this process, and this requires internal control. However, this process should be uninfluenced by the manipulation of stimulus availability. The availability heuristic may incorporate the influence of the external environment. When the stimulus affording a particular task appears, it may increase the availability of that task set. Arrington and Logan (2005) suggested that these two heuristics may function in parallel, with the more rapid availability heuristic having a greater influence at shorter RSIs. The results from Experiment 2, showing a larger effect of stimulus availability at the shorter RSI, would support this view.
The account of task choice based on competing heuristics is a useful verbal description of the cognitive processes involved in determining task choice in voluntary task switching. Ideally, however, a formal model of these processes should be developed, in which the relationship between internal and external factors is clearly specified. One candidate theory that may accommodate the present findings is ECTVA (Logan \& Gordon, 2001). As noted above, both the external evidence for a categorization, $\eta$, and the internal bias toward making that categorization, $\beta$, are key parameters in defining the task set and in guiding task performance. Consideration of the process by which these parameters are set and how they determine the choice process may increase understanding of the mechanisms that underlie the present results. First, with regard to the basic stimulus availability effect, the appearance of the stimulus establishes the $\eta$ value for that stimulus and categorization can begin. The onset of S1 initiates the race for the categorization of S1, and when the SOA is greater than 0 , the race for the categorization of $\mathrm{S} 1$ begins earlier than that for S2. Thus, the likelihood that the response produced on that trial will be appropriate for the task associated with that $\mathrm{S} 1$ is increased, resulting in higher $p(\mathrm{~S} 1)$ values. Whereas the $\eta$ values result from the external stimulus environment, other parameters within the task set, including the $\beta$ values, are under the control of the executive. Once the executive has transmitted parameters to the subordinate systems, $\beta$ values are set high for one task and low for the other task. Because the $\beta$ and $\eta$ values combine in a multiplicative fashion, high $\beta$ values for one task will drive responding when both stimuli are present. According to ECTVA, the transmission of control parameters from the executive takes time. Thus, as the RSI increases, the proportion of trials on which the $\beta$ values are set prior to the onset of either stimulus will increase. In this situation, the control parameters set by the executive may overcome the benefit of early onset of the categorization process for S1. In this way, the mechanisms of attention and categorization formalized in ECTVA may account for the relationship between stimulus availability and preparation time seen in the present research.

Other evidence also suggests an important role for basic visual attention processes in behavior in voluntary task switching environments. In a recent study of the neural basis of voluntary task switching, Forstmann, Brass, Koch, and von Cramon (2006) found evidence for a frontoparietal network differentially engaged in task choice over no-choice conditions. They interpreted the more anterior region of activation in the area of the midcingulate cortex as associated with the voluntary selection of a task set through a resolution of decision-making conflict, and the more posterior regions of activation in the superior parietal lobule and inferior parietal sulcus as associated with visual attentional selection processes. The importance of the visual attentional selection processes in performance in the task choice conditions raises an interesting possible interpretation of the present behavioral results. The onset of S1 may exogenously engage visual attention processes (Yantis \& Jonides, 1990) and thus in a bottom-up fashion influence key processes necessary for performance in the 
multitask environment through interactions of the anterior and posterior attention systems (Posner \& Petersen, 1990). Processes of visual attention may serve to mediate the influence of environmental stimuli on task choice in multitask environments. Indeed, evidence for this interpretation can be found in a voluntary task switching study incorporating hierarchical stimuli (i.e., stimuli with local and global features) with lateralized visual field presentation, in which subjects' choice of task was influenced by the visual field of presentation (Arrington \& Rhodes, 2007).

Finally, the present experiments use manipulations of timing between stimulus presentations and between trials to examine external and internal factors influencing task choice. These simple manipulations are appealing, in part because they provide an easy avenue for parametric variations in these factors. There would be clear boundary conditions beyond which such manipulations would lose their utility. If the SOA was very long, then the external factor of stimulus availability would completely drive task performance, thus removing the possibility of top-down choice. However, within reasonable values, these easily controlled parameters may provide a diagnostic tool sensitive to varying relationships between internal and external factors. For example, the relationship between task choice under varying degrees of external stimulus availability and individual differences in executive control processes, as measured by working memory span tasks (Kane \& Engle, 2003) or the attention networks test (Fan, McCandliss, Sommer, Raz, \& Posner, 2002), may provide insight into how internal and external factors interact. Such future research will be important in addressing the larger question of how cognitive control is employed in selecting and executing voluntary behavior in multitask environments.

\section{AUTHOR NOTE}

This research was partially supported by a Paul J. Franz and Class of 1968 Junior Faculty Fellowship from Lehigh University. I thank Stefanie Forlenza for her help with data collection, Karin Butler and Starla Weaver for numerous discussions throughout the course of this project, and Michael Masson, Ulrich Mayr, and several anonymous reviewers for thoughtful comments on earlier drafts of the manuscript. Correspondence concerning this article should be addressed to C. M. Arrington, Department of Psychology, Lehigh University, 17 Memorial Drive East, Bethlehem, PA 18015 (e-mail: kate.arrington(a)lehigh.edu).

\section{REFERENCES}

Allport, A., \& Wylie, G. (2000). Task switching, stimulus-response bindings, and negative priming. In S. Monsell \& J. Driver (Eds.), Control of cognitive processes: Attention and performance XVIII (pp. 35-70). Cambridge, MA: MIT Press.

Altmann, E. M. (2004). The preparation effect in task switching: Carryover of SOA. Memory \& Cognition, 32, 153-163.

Arrington, C. M., \& Logan, G. D. (2004). The cost of a voluntary task switch. Psychological Science, 15, 610-615.

Arrington, C. M., \& Logan, G. D. (2005). Voluntary task switching: Chasing the elusive homunculus. Journal of Experimental Psychology: Learning, Memory, \& Cognition, 31, 683-702.

Arrington, C. M., \& Rhodes, K. M. (2007). Perceptual asymmetries influence task choice: The effect of lateralized presentation of hierarchical stimuli. Manuscript submitted for publication.

Baddeley, A. [D.], Chincotta, D., \& Adlam, A. (2001). Working memory and the control of action: Evidence from task switching. Journal of Experimental Psychology: General, 130, 641-657.

Fan, J., McCandliss, B. D., Sommer, T., Raz, A., \& Posner, M. I.
(2002). Testing the efficiency and independence of attentional networks. Journal of Cognitive Neuroscience, 14, 340-347.

Folk, C. L., Remington, R. W., \& Johnston, J. C. (1992). Involuntary covert orienting is contingent on attentional control settings. Journal of Experimental Psychology: Human Perception \& Performance, 18, 1030-1044.

Forstmann, B. U., Brass, M., Koch, I., \& von Cramon, D. Y. (2006). Voluntary selection of task sets revealed by functional magnetic resonance imaging. Journal of Cognitive Neuroscience, 18, 388-398.

KANE, M. J., \& ENGLE, R. W. (2003). Working-memory capacity and the control of attention: The contributions of goal neglect, response competition, and task set to Stroop interference. Journal of Experimental Psychology: General, 132, 47-70.

LoGAN, G. D. (2003). Executive control of thought and action: In search of the wild homunculus. Current Directions in Psychological Science, 12, 45-48.

Logan, G. D., \& Gordon, R. D. (2001). Executive control of visual attention in dual-task situations. Psychological Review, 108, 393-434.

MAYr, U., \& Bell, T. (2006). On how to be unpredictable: Evidence from the voluntary task-switching paradigm. Psychological Science, 17, 774-780.

MeIRAN, N. (1996). Reconfiguration of processing mode prior to task performance. Journal of Experimental Psychology: Learning, Memory, \& Cognition, 22, 1423-1442.

Monsell, S. (2003). Task switching. Trends in Cognitive Sciences, 7 , 134-140.

Norman, D. A., \& Shallice, T. (1986). Attention to action: Willed and automatic control of behavior. In R. J. Davidson, G. E. Schwartz, \& D. Shapiro (Eds.), Consciousness and self-regulation: Advances in research and theory (Vol. 4, pp. 1-18). New York: Plenum.

Posner, M. I., \& Petersen, S. E. (1990). The attention system of the human brain. Annual Review of Neuroscience, 13, 25-42.

RAPOPORT, A., \& BudESCU, D. V. (1997). Randomization in individual choice behavior. Psychological Review, 104, 603-617.

Rogers, R. D., \& Monsell, S. (1995). Costs of a predictable switch between simple cognitive tasks. Journal of Experimental Psychology: General, 124, 207-231.

Ruthruff, E., Remington, R. W., \& Johnston, J. C. (2001). Switching between simple cognitive tasks: The interaction of top-down and bottom-up factors. Journal of Experimental Psychology: Human Perception \& Performance, 27, 1404-1419.

Waszak, F., Hommel, B., \& Allport, A. (2003). Task-switching and long-term priming: Role of episodic stimulus-task bindings in taskshift costs. Cognitive Psychology, 46, 361-413.

YANTIS, S., \& JonidES, J. (1990). Abrupt visual onsets and selective attention: Voluntary versus automatic allocation. Journal of Experimental Psychology: Human Perception \& Performance, 16, 121-134.

YeUnG, N., \& MoNSELL, S. (2003). Switching between tasks of unequal familiarity: The role of stimulus-attribute and response-set selection. Journal of Experimental Psychology: Human Perception \& Performance, 29, 455-469.

\section{NOTE}

1. In this and the following experiment, the proportion of task transitions was not analyzed as a function of the SOA. The task transition is related by definition to the task chosen on given trials (trial $n$ and trial $n-1)$. Thus, the influence of stimulus availability on task choice on each trial, which is the primary measure of interest, will influence the task transition probabilities as well. The designation of the letter and number stimuli as S1 and S2 on each trial is randomized by the experimenter. Therefore, the increasing effect of stimulus availability on task choice as SOA increases acts to moderate any task transition effects that might result from changes in SOA. That is, as subjects more often choose to perform the task associated with $\mathrm{S} 1$, the task transitions will consequently become more random; however, it is difficult to interpret whether such a trend toward more equal numbers of task repetitions and task switches would simply result from more random task ordering associated with the random assignment of stimuli to the $\mathrm{S} 1$ and $\mathrm{S} 2$ positions.

(Manuscript received September 6, 2007; revision accepted for publication January 14, 2008.) 